\title{
RECONSTRUCTION FROM RADON PROJECTIONS AND ORTHOGONAL EXPANSION ON A BALL
}

\author{
YUAN XU
}

\begin{abstract}
The relation between Radon transform and orthogonal expansions of a function on the unit ball in $\mathbb{R}^{d}$ is exploited. A compact formula for the partial sums of the expansion is given in terms of the Radon transform, which leads to algorithms for image reconstruction from Radon data. The relation between orthogonal expansion and the singular value decomposition of the Radon transform is also exploited.
\end{abstract}

\section{INTRODUCTION}

Reconstruction of an image from its Radon projections is the central theme in $\mathrm{x}$-ray tomography and has spectacular applications in medical imaging. Mathematically the problem is to find a good approximation to a function based on a finite collection of its Radon projections (see, for example, [9, 10, 19]).

The main topic of this paper is the connection between the Radon transform and the orthogonal expansion of the function on a unit ball. This connection was initiated in the classical paper [4] with an inversion formula of the Radon transform based on spherical harmonic expansions. The relation between the Radon transform of a function, supported on the unit ball, and its orthogonal expansion was studied or used in $[5,6,11,12,15,17]$, among others (see [19] for further references). The papers $[5,6,12]$ studied also the singular value decomposition (SVD) of the Radon transform using an orthogonal basis. Since then SVD has become an important tool for studying the stability of the inversion problem, the resolution of the reconstruction, and the incomplete data problem; see, for example, $[3,6,13,14,19]$. The truncated SVD also provides an algorithm for reconstruction of images. Because of the complicated formulas involved in the orthogonal or SVD expansions (see, for example, $[5,12,19]$ ), the algorithms did not seem to be used in practical applications.

Recently a new reconstruction algorithm was proposed in [27] and further studied in $[28,29]$. The new algorithm is called OPED, as it is based on orthogonal polynomial expansion on the unit disk. The algorithm reproduces polynomials of high degrees and allows a fast implementation ([28]). The numerical tests shows that the algorithm is fast, stable, and produces high quality images $([28,29]$. The key ingredient for deriving the algorithm is the following formula for the partial

Date: October 30, 2018.

1991 Mathematics Subject Classification. 42A38, 42B08, 42B15.

Key words and phrases. Radon transform, orthogonal expansion, reconstruction of images, algorithms.

The author was partially supported by the National Science Foundation under Grant DMS0604056 . 
sum $S_{2 m} f$ of the orthogonal expansion of $f$ on the unit disk,

$$
S_{2 m} f(x, y)=\frac{1}{2 m+1} \sum_{\nu=0}^{2 m} \int_{-1}^{1} \mathcal{R}_{\phi_{\nu}} f(t) \Phi_{2 m}\left(t, x \cos \phi_{\nu}+y \sin \phi_{\nu}\right) d t,
$$

where $\phi_{\nu}=\frac{2 \nu \pi}{2 m+1}$ and $\mathcal{R}_{\theta} f(t)$ is the Radon projection on the line $x \cos \theta+y \sin \theta=t$ (see Section 3). It turns out that there is a natural extension of this formula to the unit ball of higher dimension, which shows that the orthogonal polynomial expansion of $f$ can be expressed in terms of the Radon transforms and allows us to extend the OPED algorithm in the unit ball of $\mathbb{R}^{d}$. Furthermore, there is a close relation between SVD and the extension of the formula (1.1). In fact, they can be brought together by the use of a compact formula of the reproducing kernel of orthogonal polynomials in [24]. The orthogonal expansion on the unit ball has been studied recently in $[24,26]$, which can be used, in particular, to derive the uniform convergence of the algorithms.

The purpose of this paper is two folds. Firstly we will clarify the relation between orthogonal expansion on the ball and the Radon projections and derive the extension of the OPED algorithm in higher dimensions. Secondly, we will explain the connection between SVD of the Radon transform and orthogonal expansions. In particular, we shall show that using truncated SVD to reconstruct the image is the same as using OPED algorithm.

The paper is organized as follows. The following section contains a succinct account of the basic results on orthogonal polynomials on the unit ball. The orthogonal expansions in terms of the Radon projections is developed in Section 3. The extension of the OPED algorithms and a convergence result are given in Section 4. Finally, the SVD of the Radon transform is discussed in Section 5.

\section{Preliminaries on ORThogonal POLYNOMials}

Let $B^{d}:=\{x:\|x\| \leq 1\}$ and $S^{d-1}:=\{x:\|x\|=1\}$ be the unit ball and the unit sphere of $\mathbb{R}^{d}$, respectively. We denote the surface area of $S^{d-1}$ by $\sigma_{d}$ and the volume of $B^{d}$ by $b_{d}$. Then

$$
\sigma_{d}=\frac{2 \pi^{d / 2}}{\Gamma(d / 2)} \quad \text { and } \quad b_{d}=\frac{\sigma_{d}}{d}=\frac{\pi^{d / 2}}{\Gamma((d+2) / 2)} .
$$

Inner product on the ball. For later discussion let us introduce a weight function $W_{\mu}$ on the unit ball,

$$
W_{\mu}(x)=\left(1-\|x\|^{2}\right)^{\mu-1 / 2}, \quad x \in B^{d} .
$$

The inner product on the unit ball is denoted by

$$
\langle f, g\rangle_{L^{2}\left(B^{d}\right)}=a_{\mu} \int_{B^{d}} f(x) g(x) W_{\mu}(x) d x
$$

where $a_{\mu}$ is the normalization constant of $W_{\mu}$, that is, $a_{\mu}=1 / \int_{B^{d}} W_{\mu}(x) d x$. For $\mu=1 / 2$, it is the unit weight (Lebesgue measure) and $a_{\mu}$ is equal to $b_{d}^{-1}$. We will mainly work with the Lebesgue measure, so the inner product $\langle f, g\rangle_{L^{2}\left(B^{d}\right)}$ should be regarded as with $\mu=1 / 2$ unless specified otherwise.

Polynomial spaces. Let $\Pi_{n}^{d}$ denote the space of polynomials of degree $n$ in $d$ variables. We say that $P \in \Pi_{n}^{d}$ is an orthogonal polynomial on $B^{d}$ if $\langle P, Q\rangle_{L^{2}\left(B^{d}\right)}=$ 
0 for all $Q \in \Pi_{n-1}^{d}$. Let $\mathcal{V}_{n}^{d}$ denote the space of orthogonal polynomials. It is wellknown that

$$
\operatorname{dim} \Pi_{n}^{d}=\left(\begin{array}{c}
n+d \\
n
\end{array}\right) \quad \text { and } \quad \operatorname{dim} \mathcal{V}_{n}^{d}=\left(\begin{array}{c}
n+d-1 \\
n
\end{array}\right)
$$

Several explicit orthonormal bases of $\mathcal{V}_{n}^{d}$ are known (see, for example, [7]). We will need one given in terms of the Jacobi polynomials and spherical harmonics.

Jacobi polynomials. The $k$-th Jacobi polynomial is dentoed by $P_{k}^{(\alpha, \beta)}$ and they satisfy the orthogonal relation $([23])$

$$
\begin{aligned}
c_{\alpha, \beta} \int_{-1}^{1} P_{k}^{(\alpha, \beta)}(t) P_{l}^{(\alpha, \beta)}(t) w_{\alpha, \beta}(t) d t \\
\quad=\frac{(\alpha+1)_{k}(\beta+1)_{k}(\alpha+\beta+k+1)}{k !(\alpha+\beta+2)_{k}(\alpha+\beta+2 k+1)} \delta_{k, l}:=h_{k}^{(\alpha, \beta)} \delta_{k, l}
\end{aligned}
$$

where $w_{\alpha, \beta}(t)=(1-t)^{\alpha}(1+t)^{\beta}, c_{\alpha, \beta}$ is the normalization constant of $w_{\alpha, \beta}$,

$$
\left[c_{\alpha, \beta}\right]^{-1}=\int_{-1}^{1} w_{\alpha, \beta}(t) d t=2^{\alpha+\beta+1} \frac{\Gamma(\alpha+1) \Gamma(\beta+1)}{\Gamma(\alpha+\beta+2)},
$$

and the notation $(a)_{k}:=a(a+1) \cdots(a+k-1)$ denotes the shifted factorial (Pochhammer symbol). From (2.1) the orthonormal Jacobi polynomials are given by $p_{n}^{(\alpha, \beta)}(t):=\left[h_{n}^{(\alpha, \beta)}\right]^{-1 / 2} P_{n}^{(\alpha, \beta)}(t)$.

Gegenbauer polynomials and Chebyshev polynomials. When $\alpha=\beta=\lambda-1 / 2$, the Jacobi polynomials become the Gegenbauer polynomials, usually denoted by $C_{k}^{\lambda}$ and normalized by

$$
c_{\lambda} \int_{-1}^{1} C_{k}^{\lambda}(t) C_{l}^{\lambda}(t)\left(1-t^{2}\right)^{\lambda-1 / 2} d t=\frac{\lambda(2 \lambda)_{k}}{(k+\lambda) k !} \delta_{k, l}:=h_{k}^{(\lambda)} \delta_{k, l} .
$$

where $c_{\lambda}=\Gamma(1 / 2) \Gamma(\lambda+1 / 2) / \Gamma(\lambda+1)$. When $\lambda=1$ and $\lambda=0, C_{k}^{\lambda}(t)$ becomes the Chebyshev polynomial of the second kind, $U_{k}(t)$, and the first kind, $T_{k}(t)$, respectively, and

$$
U_{k}(t)=\frac{\sin (k+1) \theta}{\sin \theta} \quad \text { and } \quad T_{k}(t)=\cos k \theta, \quad \text { where } \quad t=\cos \theta .
$$

Spherical harmonics. These are defined as the restriction of the homogeneous harmonic polynomials on the sphere. Let $\mathcal{H}_{n}^{d}$ denote the space of spherical harmonics of degree $n$ in $d$ variables. It is known that

$$
\operatorname{dim} \mathcal{H}_{n}^{d}=\left(\begin{array}{c}
n+d-1 \\
n
\end{array}\right)-\left(\begin{array}{c}
n+d-3 \\
n
\end{array}\right) .
$$

Let $\left\{Y_{k, n}: 1 \leq k \leq \operatorname{dim} \mathcal{H}_{n}^{d}\right\}$ denote an orthonormal basis of $\mathcal{H}_{n}^{d}$. Then

$$
\sigma_{d}^{-1} \int_{S^{d-1}} Y_{k, n}(\xi) Y_{l, n}(\xi) d \omega(\xi)=\delta_{k, l}, \quad 1 \leq k, l \leq \operatorname{dim} \mathcal{H}_{n}^{d} .
$$

We emphasis that $Y_{k, n}(x)$ are in fact homogeneous polynomials in $\Pi_{n}^{d}$.

An orthonormal basis for $\mathcal{V}_{n}^{d}$. We give the basis for inner product defined in terms of $W_{\mu}(x)$. Setting $\mu=1 / 2$ gives the basis for the Lebesgue measure. Let 
$Y_{j, m}$ be an orthonormal basis for $\mathcal{H}_{m}^{d}$. Define

$$
f_{k, j}^{n}(x)=\left[h_{n, k}\right]^{-1} p_{k}^{\left(\mu-\frac{1}{2}, n-2 k+\frac{d-2}{2}\right)}\left(2\|x\|^{2}-1\right) Y_{j, n-2 k}(x),
$$

where

$$
\left[h_{n, k}\right]^{2}:=\frac{\Gamma\left(\mu+\frac{d+1}{2}\right) \Gamma\left(n-2 k+\frac{d}{2}\right)}{\Gamma\left(\frac{d}{2}\right) \Gamma\left(n-2 k+\mu+\frac{d+1}{2}\right)} .
$$

Then the set $\left\{f_{k, j}^{n}: 1 \leq j \leq \operatorname{dim} \mathcal{H}_{n-2 k}^{d}, 0 \leq 2 k \leq n\right\}$ is an orthonormal basis for $\mathcal{V}_{n}^{d}$; that is, $f_{k, j}^{n} \in \mathcal{V}_{n}^{d}$ and $\left\langle f_{k, j}^{n}, f_{k^{\prime}, j^{\prime}}^{n}\right\rangle_{L^{2}\left(B^{d}\right)}=\delta_{k, k^{\prime}} \delta_{j, j^{\prime}}$ (see [7, p. 39]).

Reproducing kernel of $\mathcal{V}_{n}^{d}$. The reproducing kernel $P_{n}(\cdot, \cdot)$ of $\mathcal{V}_{n}^{d}$ satisfies

$$
a_{\mu} \int_{B^{d}} f(y) P_{n}(x, y) W_{\mu}(y) d y=f(x), \quad \forall f \in \mathcal{V}_{n}^{d} .
$$

Let $\left\{P_{k}^{n}: 1 \leq k \leq \operatorname{dim} \mathcal{V}_{n}^{d}\right\}$ denote any orthonormal basis of $\mathcal{V}_{n}^{d}$. Then

$$
P_{n}(x, y)=\sum_{k=1}^{N_{n}} P_{k}^{n}(x) P_{k}^{n}(y), \quad N_{n}=\operatorname{dim} \mathcal{V}_{n}^{d} .
$$

The definition of $P_{n}(\cdot, \cdot)$, however, is independent of the particular choice of bases. In particular, we can take the orthonormal basis in (2.6) and get

$$
P_{n}(x, y)=\sum_{0 \leq 2 k \leq n} \sum_{j=1}^{\operatorname{dim} \mathcal{H}_{n-2 k}^{d}} f_{k, j}^{n}(x) f_{k, j}^{n}(y) .
$$

The reproducing kernel satisfies a compact formula that will play a fundamental role in our study; it is given by $([24])$

$$
P_{n}(x, y)=\frac{n+\lambda}{\lambda} c_{\mu-\frac{1}{2}} \int_{-1}^{1} C_{n}^{\lambda}\left(\langle x, y\rangle+\sqrt{1-\|x\|^{2}} \sqrt{1-\|y\|^{2}} s\right)\left(1-s^{2}\right)^{\mu-1} d s
$$

where $\lambda=\mu+\frac{d-1}{2},\langle\cdot, \cdot\rangle$ is the Euclidean inner product in $\mathbb{R}^{d}$, and $c_{\lambda}$ is defined in (2.2). In particular, it implies that

$$
P_{n}(x, \xi)=\frac{n+\lambda}{\lambda} C_{n}^{\lambda}(\langle x, \xi\rangle), \quad \xi \in S^{d-1}, \quad x \in B^{d} .
$$

Orthogonal expansions on $B^{d}$. If $\left\{P_{k}^{n}: 1 \leq k \leq N_{n}\right\}, N_{n}=\operatorname{dim} \mathcal{V}_{n}^{d}$, is an orthonormal basis of $\mathcal{V}_{n}^{d}$, then the standard Hilbert space theory states that there is an orthogonal expansion

$$
f=\sum_{k=0}^{\infty} \sum_{k=1}^{N_{n}}\left\langle f, P_{k}^{n}\right\rangle_{L^{2}\left(B^{d}\right)} P_{k}^{n}, \quad \forall f \in L^{2}\left(B^{d}\right) .
$$

Let $\operatorname{proj}_{k}: L^{2}\left(B^{d}\right) \mapsto \mathcal{V}_{n}^{d}$ denote the projection operator. Using the reproducing kernel, the orthogonal expansion can be stated as

$$
f=\sum_{k=0}^{\infty} \operatorname{proj}_{k} f, \quad \text { where } \quad \operatorname{proj}_{k} f=a_{\mu} \int_{B^{d}} f(y) P_{n}(x, y) W_{\mu}(y) d y,
$$

which is independent of the particular choices of the bases of $\mathcal{V}_{n}^{d}$. 


\section{Radon Transform and Orthogonal Polynomial Expansion}

Let $f \in L^{1}$ be a real valued function. For $\xi \in S^{d-1}$ and $t \in \mathbb{R}$, the Radon transform of $f$ is defined as

$$
\mathcal{R} f(\xi, t):=\int_{\langle\xi, x\rangle=t} f(x) d x=\int_{\xi^{\perp}} f(t \xi+y) d y,
$$

where the integral is over a hyperplane of $(d-1)$-dimension perpendicular to $\xi$ and with minimum distance $t$ to the origin. More general definition on other spaces or manifolds can be found in [8]. For properties of Radon transforms we refer to $[8,19]$. We assume that $f$ has compact support in $B^{d}$, so that the integral above should be understood as over $B^{d} \cap\{x:\langle\xi, x\rangle=t\}$. In particular, for $\xi \in S^{d-1}$, let $Q_{\xi}$ denote an orthogonal matrix whose first row is $\xi$ and let $B^{d}(r)$ denote the ball of radius $r$ in $\mathbb{R}^{d}$; then a change of variables $x \mapsto(t, y) Q_{\xi}$ shows that

$$
\begin{aligned}
\mathcal{R} f(\xi, t) & =\int_{B^{d-1}\left(\sqrt{1-t^{2}}\right)} f\left((t, y) Q_{\xi}\right) d y \\
& =\left(1-t^{2}\right)^{\frac{d-1}{2}} \int_{B^{d-1}} f\left(\left(t, \sqrt{1-t^{2}} y\right) Q_{\xi}\right) d y .
\end{aligned}
$$

Since $\left\langle(t, y) Q_{\xi}, \xi\right\rangle=t$, an immediate consequence of (3.1) is the following identity,

$$
\int_{B^{d}} f(x) g(\langle x, \xi\rangle) d x=\int_{-1}^{1} \mathcal{R} f(\xi, t) g(t) d t, \quad \xi \in S^{d-1}
$$

whenever both integrals make sense. The definition of $\mathcal{R} f$ also implies that

$$
\mathcal{R} f(-\xi,-t)=\mathcal{R} f(\xi, t), \quad \xi \in S^{d-1}, \quad t \in \mathbb{R} .
$$

For fixed $\xi$ and $t$, we also call $\mathcal{R} f(\xi, t)$ a Radon projection. The essential problem for x-ray imaging is to find a good approximation to the function $f$ based on a given data set of its Rdaon projections.

We now derive the orthogonal expansion of $f$ in terms of Radon projections. The following proposition plays a key role.

Proposition 3.1. For $x, y \in B^{d}$, the reproducing kernel $P_{n}(\cdot, \cdot)$ satisfies

$$
P_{n}(x, y)=\frac{n+d / 2}{d / 2} \sigma_{d}^{-1} \int_{S^{d-1}} C_{n}^{d / 2}(\langle x, \xi\rangle) C_{n}^{d / 2}(\langle y, \xi\rangle) d \omega(\xi) .
$$

Proof. From the explicit formula of $f_{k, j}^{n}$ at (2.4) with $\mu=1 / 2$, we deduce that

$$
f_{k, j}^{n}(\xi)=H_{n} Y_{j, n-2 k}(\xi), \quad \xi \in S^{d-1},
$$

where, using the fact that $p_{k}^{(0, \beta)}(t)=\left[h_{k}^{(0, \beta)}\right]^{-1 / 2} P_{k}^{(0, \beta)}(t), P_{k}^{(0, \beta)}(1)=1$, and the formula of $h_{k}^{(\alpha, \beta)}$ in (2.1), we have

$$
H_{n}=\left[h_{n, k}\right]^{-1} p_{k}^{\left(0, n-2 k+\frac{d-2}{2}\right)}(1)=\sqrt{\frac{n+d / 2}{d / 2}},
$$

independent of $k$. Consequently, integrating over $S^{d-1}$ we get

$$
\sigma_{d}^{-1} \int_{S^{d-1}} f_{k, j}^{n}(\xi) f_{k^{\prime}, j^{\prime}}^{n}(\xi) d \omega(\xi)=H_{n}^{2} \delta_{j, j^{\prime}} \delta_{k, k^{\prime}}=\frac{n+d / 2}{d / 2} \delta_{j, j^{\prime}} \delta_{k, k^{\prime}}
$$

Multiplying the above equation by $f_{k, j}^{n}(x)$ and $f_{k^{\prime}, j^{\prime}}^{n}(y)$ and summing over all $j, j^{\prime}, k, k^{\prime}$, the stated result follows from (2.6) and (2.7). 
Theorem 3.2. For $n \geq 0$,

$$
\operatorname{proj}_{n} f(x)=\frac{n+d / 2}{d / 2} \sigma_{d}^{-1} \int_{S^{d-1}} b_{d}^{-1} \int_{-1}^{1} \mathcal{R} f(\xi, t) C_{n}^{d / 2}(t) d t C_{n}^{d / 2}(\langle x, \xi\rangle) d \omega(\xi) .
$$

In particular, for $f \in L^{2}\left(B^{d}\right)$,

$$
f=\sum_{n=0}^{\infty} \frac{n+d / 2}{d / 2} \sigma_{d}^{-1} \int_{S^{d-1}} b_{d}^{-1} \int_{-1}^{1} \mathcal{R} f(\xi, t) C_{n}^{d / 2}(t) d t C_{n}^{d / 2}(\langle\cdot, \xi\rangle) d \omega(\xi) .
$$

Proof. By the formula (2.5) with $\mu=1 / 2$ and the formula (3.4) of $P_{n}(\cdot, \cdot)$ we have

$$
\begin{aligned}
\operatorname{proj}_{n} f(x) & =b_{d}^{-1} \int_{B^{d}} f(y) P_{n}(x, y) d y \\
& =\frac{n+d / 2}{d / 2} \sigma_{d}^{-1} \int_{S^{d-1}} b_{d}^{-1} \int_{B^{d}} f(y) C_{n}^{d / 2}(\langle y, \xi\rangle) d y C_{n}^{d / 2}(\langle x, \xi\rangle) d \omega(\xi) .
\end{aligned}
$$

The identity (3.2) shows that the inner integral is

$$
\int_{B^{d}} f(y) C_{n}^{d / 2}(\langle y, \xi\rangle) d y=\int_{-1}^{1} \mathcal{R} f(\xi, t) C_{n}^{d / 2}(t) d t
$$

so that the stated formula follows.

The formula (3.7) as stated here has already appeared in [20] in a study of the approximation by ridge functions. See also [1] for the case of $d=2$. Although spherical harmonics expansions for $d=2$ was used in the classical work of [4], its compact form in (3.7) is quite recent and not used for reconstructing images from Radon data until recently ([27]). It should also be noted that for $d>2$, the Gegenbauer polynomials and spherical harmonics were used for constructing Radon transforms already in [12].

Let us mention that there does not seem to be an analogous formula for the more general case of orthogonal expansion with respect to $W_{\mu}$. In fact, in the general case, the formula (2.4) gives

$$
f_{k, j}^{n}(\xi)=H_{n, k} Y_{j, n-2 k}(\xi), \quad \xi \in S^{d-1},
$$

where

$$
H_{n, k}:=\frac{(\mu+1 / 2)_{k}\left(\mu+\frac{d-1}{2}\right)_{n-k}\left(n+\mu+\frac{d-1}{2}\right)}{k !\left(\frac{d}{2}\right)_{n-k}\left(\mu+\frac{d-1}{2}\right)},
$$

which depends on both $n$ and $k$ (comparing with (3.6)), so that Proposition 3.1 with $C_{n}^{d / 2}$ replaced by $C_{n}^{\mu+\frac{d-1}{2}}$ does not hold.

Let $S_{n} f$ denote the partial sum operator of the orthogonal expansion (2.8),

$$
S_{n} f(x)=\sum_{k=0}^{n} \operatorname{proj}_{k} f(x) .
$$

Evidently, the expansion (2.8) holds in the sense that $S_{n} f \rightarrow f$ in $L^{2}\left(B^{d}\right)$ norm.

Corollary 3.3. Let $S_{n}$ be the partial sum operator defined in (3.10). Then

$$
S_{n} f(x)=\sigma_{d}^{-1} \int_{S^{d-1}} b_{d}^{-1} \int_{-1}^{1} \mathcal{R} f(\xi, t) \Phi_{n}(t,\langle x, \xi\rangle) d t d \omega(\xi) .
$$


where

$$
\Phi_{n}(t, u):=\sum_{k=0}^{n} \frac{k+d / 2}{d / 2} C_{k}^{d / 2}(t) C_{k}^{d / 2}(u) .
$$

A cubature formula on $S^{d-1}$ of degree $M$ is a discrete sum such that

$$
\sigma_{d}^{-1} \int_{S^{d-1}} f(\xi) d \omega(\xi)=\sum_{\nu=1}^{N} \lambda_{\nu} f\left(\xi_{\nu}\right), \quad f \in \Pi_{M}\left(S^{d-1}\right),
$$

where $\Pi_{M}\left(S^{d-1}\right)$ is the space of spherical polynomials, that is, the space of $\Pi_{M}^{d}$ restricted on $S^{d-1}$. If all $\lambda_{k}$ are positive, the cubature is called positive. We call a polynomial $P \in \Pi_{M}^{d}$ even if it satisfies $P(x)=P(-x)$ for all $x \in \mathbb{R}^{d}$. The cubature formula (3.13) is called symmetric, if it is exact for all even polynomials in $\Pi_{M}\left(S^{d-1}\right)$.

Proposition 3.4. Suppose (3.13) is a symmetric cubature formula of degree $2 n$. Then

$$
S_{n} f(x)=\sum_{\nu=1}^{N} \lambda_{\nu} b_{d}^{-1} \int_{-1}^{1} \mathcal{R} f\left(\xi_{\nu}, t\right) \Phi_{n}\left(t,\left\langle x, \xi_{\nu}\right\rangle\right) d t
$$

Proof. The equation (3.8) shows that $P_{x}(\xi):=\int_{-1}^{1} \mathcal{R} f(\xi, t) \Phi_{n}(\xi, t ; x) d t$ is a polynomial of degree at most $2 n$ in $\xi$. Furthermore, using the fact that $\mathcal{R} f(-\xi,-t)=$ $\mathcal{R} f(\xi, t)$, it is easy to see that $P_{x}$ is even, so that the cubature formula on $S^{d-1}$ is exact when applied to $P_{x}(\xi)$.

We consider some special cases of lower dimensions below.

The case $\mathbf{d}=\mathbf{2}$. For $\xi \in S^{1}$ we write $\xi=(\cos \theta, \sin \theta)$ and we shall write $\mathcal{R}_{\theta} f(t)$, $\theta \in[0,2 \pi]$, instead of $\mathcal{R} f(\xi, t)$. Since $b_{2}=\pi$ and the following cubature formula

$$
\frac{1}{2 \pi} \int_{S^{1}} f(\xi) d \omega(\xi)=\frac{1}{n+1} \sum_{\nu=0}^{n} f\left(\xi_{\nu}\right), \quad \xi_{\nu}=\left(\cos \frac{\nu \pi}{n+1}, \sin \frac{\nu \pi}{n+1}\right)
$$

is symmetric and of degree $2 n$, we conclude that

$$
S_{n} f(x)=\frac{1}{n+1} \sum_{\nu=0}^{n} \int_{-1}^{1} \mathcal{R}_{\theta_{\nu}} f(t) \Phi_{n}\left(t, x_{1} \cos \theta_{\nu}+x_{2} \sin \theta_{\nu}\right) d t
$$

where $\theta_{\nu}=\frac{\nu \pi}{n+1}$ and $\Phi_{n}$ is $(3.12)$ for $d=2$,

$$
\Phi_{n}(t, u)=\sum_{k=0}^{n}(k+1) U_{k}(t) U_{k}(u)
$$

This formula can be found implicitly in [11] (see (5.9), (4.3) and (3.7) there). In the case of $n=2 m$, we can use the elementary relations

$$
\cos \frac{(2 \nu+1) \pi}{2 m+1}=-\cos \frac{(2(\nu+m) \pi}{2 m+1}, \quad \sin \frac{(2 \nu+1) \pi}{2 m+1}=-\sin \frac{(2(\nu+m) \pi}{2 m+1}
$$

and the fact that $\mathcal{R}(\theta+\pi,-t)=\mathcal{R}(\theta, t)$ to rewrite $(3.15)$ as

$$
S_{2 m} f(x)=\frac{1}{2 m+1} \sum_{\nu=0}^{2 m} \int_{-1}^{1} \mathcal{R}_{\phi_{\nu}} f(t) \Phi_{2 m}\left(t, x_{1} \cos \phi_{\nu}+x_{2} \sin \phi_{\nu}\right) d t
$$


where $\phi_{\nu}=\frac{2 \nu \pi}{2 m+1}$. This is the formula (1.1) proved in [27] from which the OPED algorithms are derived.

The case $\mathbf{d}=\mathbf{3}$. For $\xi \in S^{2}$ we use the spherical coordinate

$$
\xi=(\sin \phi \sin \theta, \sin \phi \cos \theta, \cos \phi), \quad 0 \leq \phi \leq \pi, 0 \leq \theta \leq 2 \pi .
$$

Several explicit cubature formulas on the sphere are known, see, for example, $[18$, 21 . Let $t_{k}=\cos \theta_{k}, k=0,1, \ldots, n$, denote the zeros of the Legendre polynomial of degree $n+1$ and $\lambda_{k}$ be the corresponding weights of the Legendre-Gaussian quadrature formula. Let

$$
\xi_{k, \nu}=\left(\sin \frac{\nu \pi}{n+1} \sin \theta_{k}, \cos \frac{\nu}{n+1} \sin \theta_{k}, \cos \theta_{k}\right), \quad 0 \leq k, \nu \leq n
$$

Then the product type cubature formula

$$
\frac{1}{4 \pi} \int_{S^{2}} f(\xi) d \omega(\xi)=\frac{1}{n+1} \sum_{k=0}^{n} \lambda_{k} \sum_{\nu=0}^{n} f\left(\xi_{k, \nu}\right)
$$

is symmetric and of degree $2 n$. Consequently, we have

$$
S_{n} f(x)=\frac{1}{n+1} \sum_{k=0}^{n} \lambda_{k} \sum_{\nu=0}^{n} \int_{-1}^{1} \mathcal{R} f\left(\xi_{k, \nu}, t\right) \Phi_{n}\left(t,\left\langle\xi_{k, \nu}, x\right\rangle\right) d t,
$$

where $\Phi_{n}$ is the function (3.12) for $d=3$.

The formula of $S_{n} f$ in terms of Radon projections allows us to give an approximation to $f$ based on finite Radon projections. The convergence of $S_{n} f$ to $f$ holds in $L^{2}$ norm but does not hold in the uniform norm in general. In fact, it is known that $[25]$

$$
\left\|S_{n}\right\|_{\infty}=\mathcal{O}\left(n^{\frac{d-1}{2}}\right), \quad d \geq 2,
$$

where $\|\cdot\|_{\infty}$ is the operator norm of $S_{n}$ in $C\left(B^{d}\right)$, and $A_{n}=\mathcal{O}\left(B_{n}\right)$ means $c_{1} A_{n} \leq$ $B_{n} \leq c_{2} A_{n}$ for two constants $c_{1}$ and $c_{2}$ independent of $n$. There is, however, a simple construction that gives a better convergence result.

Let $\eta$ be a $C^{d+2}(\mathbb{R})$ function such that $\eta(t) \geq 0, \eta(t)=1$ for $0 \leq t \leq 1$ and $\eta$ has compact support on $[0,2]$. Define

$$
S_{n}^{\eta} f(x):=\sum_{k=0}^{2 n} \eta\left(\frac{k}{n}\right) \operatorname{proj}_{k} f(x) .
$$

The operator $S_{n}^{\eta}$ satisfies the following properties [26]:

Proposition 3.5. Let $f \in L^{p}\left(B^{d}\right), 1 \leq p<\infty$ or $f \in C\left(B^{d}\right)$ for $p=\infty$. Then

(1) $S_{n}^{\eta} f=f$ if $f \in \Pi_{n}$;

(2) for $n \in \mathbb{N},\left\|\eta_{n} f\right\|_{p} \leq c\|f\|_{p}$

(3) for $n \in \mathbb{N},\left\|f-\eta_{n}\right\|_{p} \leq c E_{n}(f)_{p}:=\inf _{p \in \Pi_{n}^{d}}\|f-p\|_{p}$.

As $S_{n}^{\eta} f$ is a polynomial of degree $2 n$, the last property shows that, up to a constant multiple, it is close to the polynomial of the best approximation to $f$. Since $\operatorname{proj}_{n} f$ can be written in terms of Radon projections, so can $S_{n}^{\eta} f$. 


\section{OPED ALGORITHMS FOR RECONSTRUCTION OF IMAGES}

The essential problem in computerized tomography is to find a good approximation to the function $f$ based on a set of discrete Radon data. The expression (3.14) allows us to derive such an approximation by a simple quadrature formula on $[-1,1]$. Because of $(3.1)$, we choose the quadrature formula to be of the form

$$
c_{d / 2} \int_{-1}^{1} f(t)\left(1-t^{2}\right)^{\frac{d-1}{2}} d t=\sum_{j=0}^{n} w_{j} f\left(t_{j}\right),
$$

where $c_{d / 2}$ is defined as in (2.2), and assume that it is exact for polynomials of degree $M$. In particular, we can choose the Gaussian quadrature, for which $t_{j}=t_{j, n}$, $0 \leq j \leq n$, are zeros of the Gegenbauer polynomial $C_{n+1}^{d / 2}(t)$ and $w_{j}$ are all positive and given by explicit formula (see [23]). The Gaussian quadrature formula is exact for polynomials of degree up to $2 n+1$.

Proposition 4.1. Let (3.13) be a positive symmetric cubature formula of degree $2 n$ and (4.1) be the Gaussian quadrature formula. Define

$$
\mathcal{A}_{n} f(x)=b_{d}^{-1} \sum_{\nu=1}^{N} \lambda_{\mu} \sum_{j=0}^{n} w_{j} \mathcal{R} f\left(\xi_{\nu}, t_{j}\right) \Phi_{n}\left(t_{j},\left\langle x, \xi_{\nu}\right\rangle\right) .
$$

Then $\mathcal{A}_{n} f$ preserves polynomials of degree $n$, that is, $\mathcal{A}_{n} f=f$ whenever $f \in \mathcal{P}_{n}^{d}$.

Proof. We start from (3.14). If $f$ is a polynomial of degree at most $n$ then, by (3.1), $\left(1-t^{2}\right)^{-\frac{d-1}{2}} \mathcal{R} f\left(\xi_{\nu}, t\right)$ is a polynomial of degree $n$. As $\Phi_{n}\left(t,\left\langle x, \xi_{\nu}\right\rangle\right)$ is a polynomial of degree $n$ in $t$ and the Gaussian quadrature formula is of degree $2 n+1$, the fact that $\mathcal{A}_{n} f=f$ follows.

The functions $\mathcal{A}_{n} f$ are obtained from the orthogonal partial sums $S_{n} f$ of $f$ by applying the Gaussian quadrature formula. They provide a sequence of approximation to $f$ based on the set of discrete Radon data

$$
\left\{\mathcal{R} f\left(\xi_{\nu}, t_{j}\right): \quad 1 \leq \nu \leq N, 0 \leq j \leq n\right\}
$$

In other word, $\mathcal{A}_{n}$ provides an algorithm for reconstruction of images from the Radon data. We will show that $\mathcal{A}_{n} f$ converges to $f$ uniformly if $f$ is smooth enough. First, however, we consider some special cases.

The case $\mathbf{d}=\mathbf{2}$. In this case we can start from the formula of $S_{2 m}$ at (3.16). The Gaussian quadrature formula is

$$
\frac{1}{\pi} \int_{-1}^{1} f(t) \sqrt{1-t^{2}} d t=\frac{1}{2 m+1} \sum_{j=1}^{2 m} \sin ^{2} \psi_{j} f\left(\cos \theta_{j}\right), \quad \theta_{j}=\frac{j \pi}{2 m+1},
$$

which leads to the OPED algorithm of type II,

$$
\mathcal{A}_{2 m} f(x)=\sum_{\nu=0}^{2 m} \sum_{j=1}^{2 m} \mathcal{R}_{\phi_{\nu}} f\left(\cos \theta_{j}\right) T_{j, \nu}(x),
$$

where

$$
T_{j, \nu}(x)=\frac{1}{(2 m+1)^{2}} \sum_{k=0}^{2 m}(k+1) \sin \left((k+1) \theta_{j}\right) U_{k}\left(x_{1} \cos \phi_{\nu}+x_{2} \sin \phi_{\nu}\right) .
$$


The OPED of type II is closely related to an algorithm in [2], where the connection to orthogonal polynomial expansion was not considered. The formation of the lines on which the Radon projections take place is often refereed to as scanning geometry, as it determines how the object being examined is scanned by the x-rays. We can use the Gaussian quadrature formula for the Chebyshev polynomials of the first kind,

$$
\frac{1}{\pi} \int_{-1}^{1} f(t) \frac{d t}{\sqrt{1-t^{2}}}=\frac{1}{2 m+1} \sum_{k=0}^{2 m} f\left(\cos \psi_{j}\right), \quad \psi_{j}=\frac{\left(j+\frac{1}{2}\right) \pi}{2 m+1},
$$

to discretize the integral in (3.16) by applying it to the integrant multiplied by $1-t^{2}$, leading to the OPED algorithm of type I with a different scanning geometry, which has the same formula as (4.3) except that $\theta_{j}$ need to be replaced by $\psi_{j}$ and the summation on $j$ starts from $j=0$. We refer to [29] for the discussions of these two scanning geometries and their implementation in practical problems.

Both types of these two OPED algorithms work well in our numerical testing ([28, $29])$. It should be mentioned that the explicit formula of $U_{n}(t)$ in $(2.3)$ permits a fast implementation of the OPED algorithm, which uses fast Fourier sine transform and an interpolation step ([28]).

The case $d=3$. In this case we can start from the formula of $S_{n} f$ at (3.17). We apply the Gaussian quadrature formula

$$
\frac{3}{4} \int_{-1}^{1} f(t)\left(1-t^{2}\right) d t=\sum_{j=0}^{n} w_{j} f\left(t_{j}\right)
$$

where $t_{j}, 0 \leq j \leq n$, are zeors of $C_{n}^{3 / 2}(t)$. We can also apply the Gaussian quadrature formula for the Lebesgue measure. This leads to a three dimensional OPED algorithm,

$$
\mathcal{A}_{n} f(x)=\frac{1}{n+1} \sum_{k=0}^{n} \lambda_{k} \sum_{\nu=0}^{n} \sum_{j=0}^{n} w_{j} \mathcal{R} f\left(\xi_{k, \nu}, t_{j}\right) \Phi_{n}\left(t_{j},\left\langle\xi_{k, \nu}, x\right\rangle\right) .
$$

The Radon data used in (4.4) are integrals over planes $\left\langle x, \xi_{k, \nu}\right\rangle=t_{j}$. Such data can be approximated by integrals over lines.

For $d=3$, one can uses multiple 2D slices to reconstruct image on a cylindrical domain, as proposed in [27]. An interesting question is to see which of these two algorithms are more suitable for the $3 \mathrm{D}$ reconstruction.

Next we consider the convergence of $\mathcal{A}_{n} f$ in the uniform norm on $B^{d}$.

Theorem 4.2. The uniform norm of the operator $\mathcal{A}_{n}$ is given by

$$
\|\mathcal{A}\|_{\infty}=\sup _{x \in B^{d}} \Lambda_{n}(x), \quad \Lambda_{n}(x)=\sum_{\nu=1}^{N} \lambda_{\nu} \sum_{j=0}^{n} w_{j}\left(1-t_{j}^{2}\right)^{\frac{d-1}{2}}\left|\Phi_{n}\left(t_{j},\left\langle x, \xi_{\nu}\right\rangle\right)\right| .
$$

Furthermore, there is a constant $c$ independent of $n$, such that

$$
\|\mathcal{A}\|_{\infty} \leq c n^{2 d} .
$$

In particular, if $f$ is smooth enough then $\mathcal{A}_{2 n} f$ converges to $f$ uniformly on $B^{d}$.

Proof. To estimate the norm of $\mathcal{A}_{n}$, we first observe that

$$
\left|\left(1-t^{2}\right)^{-\frac{d-1}{2}} \mathcal{R} f\left(\xi_{\nu}, t\right)\right| \leq b_{d-1}\|f\|_{\infty}
$$


from which it follows that

$$
\left\|\mathcal{A}_{n} f\right\|_{\infty} \leq\|f\|_{\infty} \sum_{\nu=1}^{N} \lambda_{\nu} \sum_{j=0}^{n} w_{j}\left(1-t_{j}^{2}\right)^{\frac{d-1}{2}}\left|\Phi_{n}\left(t_{j},\left\langle x, \xi_{\nu}\right\rangle\right)\right|,
$$

since $b_{d-1} b_{d}^{-1}=c_{d / 2}$. Taking the maximum over $B^{d}$ shows that $\|\mathcal{A}\|_{\infty}$ is bounded by the right hand side of (4.5). To prove the equal sign, we construct a function $f_{\varepsilon}$ for each $\varepsilon>0$ such that $\left\|f_{\varepsilon}\right\|_{\infty}=1$ and $\left\|\mathcal{A} f_{\varepsilon}\right\|_{\infty} \geq \max _{x \in B^{d}} \Lambda_{n}(x)-c \varepsilon$. A moment of reflection shows that the construction can be carried out easily; see [27] for one special case of $d=2$.

To prove (4.6) we use (3.12) and the fact that $\left|C_{n}^{\lambda}(t)\right| \leq C_{n}^{\lambda}(1)=\left(\begin{array}{c}n+2 \lambda-1 \\ n\end{array}\right)=$ $\mathcal{O}\left(n^{2 \lambda-1}\right)$, which implies that

$$
\left|\Phi_{n}(\xi, t)\right| \leq \sum_{k=0}^{n} \frac{k+d / 2}{d / 2}\left[C_{n}^{d / 2}(1)\right]^{2} \leq c \sum_{k=0}^{n} \frac{k+d / 2}{d / 2} k^{2 d-2} \leq c n^{2 d} .
$$

Since $\lambda_{\mu}$ and $w_{j}$ are all positive and, as the cubature and the quadrature are exactly for constant function, $\sum_{\nu=1}^{N} \lambda_{\nu}=1$ and $\sum_{j=0}^{n} w_{j}=1$, we conclude that $\left\|\mathcal{A}_{n}\right\| \leq c n^{2 d}$. If $f \in C^{2 d}$, then the fact that $\mathcal{A}_{n} p=p$ for $p \in \Pi_{n}^{d}$ and the triangle inequality shows that

$$
\left\|\mathcal{A}_{n} f-f\right\|_{\infty} \leq\left(1+\|\mathcal{A}\|_{\infty}\right) E_{n}(f)_{\infty} \leq c n^{2 d} E_{n}(f)_{\infty} .
$$

It is shown in [27] that $E_{n}(f) \leq c n^{-2 r}\left\|\mathcal{D}^{r} f\right\|$, where $\mathcal{D}$ is a second order differential operator, so that the convergence of $\mathcal{A}_{n} f$ for functions smooth enough follows.

We should point out that the estimate (4.6) is a rough upper bound, the actual norm should be smaller. In fact, in the case of $d=2$, the norm of $\mathcal{A}_{2 m}$ at (4.3) was estimated in [27] to be

$$
\left\|\mathcal{A}_{2 m}\right\|_{\infty} \sim m \log (m+1)
$$

which is sharp and is just slightly worse than the estimate (3.18) of the norm of the partial sum operator $S_{n}$ from which $\mathcal{A}_{2 m}$ is obtained. The proof of such a sharp estimate is rather involved and requires detail knowledge of the zeros and weights of the quadrature and cubature formulas. On the other hand, a result in [22] shows that the norm of any projection operator from $C\left(B^{d}\right)$ to $\Pi_{n}^{d}$ is at least $\mathcal{O}\left(n^{\frac{d-1}{2}}\right)$ for $d \geq 2$. As $\mathcal{A}_{n}$ in (4.2) is in fact a projection operator, its norm cannot be bounded. We expect that the norm is in the order of $\mathcal{O}\left(n^{d / 2}\right)$ multiplied by a log factor.

It should be mentioned that other polynomial based algorithms may have better approximation property $([15,16]$. However, the polynomial preserving property seems to be an important characteristic of OPED and using the partial sum allows also fast implementation of the algorithm. The numerical tests show that OPED works very well even for step functions such as Logan-Sheff head phantom [28, 29].

\section{Singular value decomposition of the Radon transform}

Let $A: H \mapsto K$ be a linear continuous operator, where $H$ and $K$ are Hilbert spaces. Let $\left\{f_{k}\right\}_{k>0}$ and $\left\{g_{k}\right\}_{k>0}$ be orthonormal systems with respect to the inner product $\langle\cdot, \cdot\rangle_{H}$ in $H$ and $\langle\cdot, \cdot\rangle_{K}$ in $K$, respectively. The singular value decomposition of $A$ is a representation

$$
A f=\sum_{k=1}^{\infty} \gamma_{k}\left\langle f, f_{k}\right\rangle_{H} g_{k},
$$


where $\gamma_{k}$ are the singular values of $A$. Let $A^{*}$ be the adjoint of $A$. Then

$$
A^{*} g=\sum_{k=1}^{\infty} \gamma_{k}\left\langle g, g_{k}\right\rangle_{K} f_{k}
$$

Evidently $A f_{k}=\gamma_{k} g_{k}$ and $A^{*} g_{k}=\gamma_{k} f_{k}$. Furthermore, the generalized inverse of $A$ is given by

$$
A^{+} g=\sum_{k=0}^{\infty} \gamma_{k}^{-1}\left\langle f, f_{k}\right\rangle_{H} g_{k} .
$$

The singular value decomposition of the Radon transform was developed in [5, 12] (see also [19]). Let $Z=S^{d-1} \times[-1,1]$ and $w(t)=\sqrt{1-t^{2}}$, and denote by $L^{2}\left(Z, w^{1-d}\right)$ the space of Lebesgue integrable functions

$$
L^{2}\left(Z, w^{1-d}\right):=\left\{g: g(-\xi,-t)=g(\xi, t), \quad\|g\|_{L^{2}(Z)}<\infty\right\},
$$

where $\|g\|_{L^{2}(Z)}^{2}=\langle g, g\rangle_{L^{2}(Z)}$ and the inner product is defined by

$$
\langle f, g\rangle_{L^{2}(Z)}:=c_{d / 2} \int_{-1}^{1} \sigma_{d}^{-1} \int_{S^{d-1}} f(\xi, t) g(\xi, t) d \omega(\xi)\left(1-t^{2}\right)^{\frac{1-d}{2}} d t,
$$

in which $c_{d / 2}$ is defined as in (2.2). Then it is known (see, for example, [19]) that

$$
\mathcal{R}: L^{2}\left(B^{d}\right) \mapsto L^{2}\left(Z, w^{1-d}\right)
$$

is continuous. An orthonormal basis of $L^{2}\left(Z, w^{1-d}\right)$ is readily available.

Proposition 5.1. Let $\left\{Y_{j, m}: 1 \leq j \leq \operatorname{dim} \mathcal{H}_{m}^{d}\right\}$ denote an orthogomal basis of $\mathcal{H}_{m}^{d}$ and define

$$
g_{k, j}^{n}(\xi, t)=\left[h_{n}^{(d / 2)}\right]^{-1 / 2}\left(1-t^{2}\right)^{\frac{d-1}{2}} C_{n}^{d / 2}(t) Y_{j, n-2 k}(\xi),
$$

where $h_{n}^{(d / 2)}$ is defined in (2.2). Then the functions $\left\{g_{k, j}^{n}: 0 \leq 2 k \leq n, 1 \leq j \leq\right.$ $\left.\operatorname{dim} \mathcal{H}_{n-2 k}^{d}\right\}$ forms an orthogonomral basis for $L^{2}\left(Z, w^{1-d}\right)$.

Proof. It is straightforward to verify that $\left\{g_{k, j}^{n}\right\}$ form an orthonormal system of $L^{2}\left(Z, w^{1-d}\right)$. Let $g \in L^{2}\left(Z, w^{1-d}\right)$. Then $w^{2 d-2} g$ can be expanded in terms of the product orthonomal basis $\left\{\left[h_{n}^{(d / 2)}\right]^{-1 / 2} C_{n}^{d / 2}(t) Y_{j, n-m}(\xi): 0 \leq m \leq n, 0 \leq\right.$ $\left.j \leq \operatorname{dim} \mathcal{H}_{n-m}^{d}\right\}$ of $L^{2}\left(Z, w^{d-1}\right)$. The condition $g(-\xi,-t)=g(\xi, t)$ shows that the coefficients of the expansion are zero whenever $m$ is odd, so that we can assume $m=2 k$ and the expansion is uniquely determined.

Using $f_{k, j}^{n}$ in (2.4) and $g_{k, j}^{n}(5.4)$, the singular value decomposition of the Radon transform at (5.1), (5.2) and (5.3) become the following:

Theorem 5.2. Assume $f$ is in the Schwartz space. The singular decomposition of $\mathcal{R} f$ is

$$
\mathcal{R} f=\sum_{n=0}^{\infty} \gamma_{n} \sum_{0 \leq 2 k \leq n} \sum_{j=0}^{M_{n-2 k}}\left\langle f, f_{k, j}^{n}\right\rangle_{L^{2}\left(B^{d}\right)} g_{k, j}^{n}
$$

where $M_{m}=\operatorname{dim} \mathcal{H}_{m}^{d}, c_{d / 2}$ is defined at (2.2); and

$$
\mathcal{R}^{*} g=\sum_{n=0}^{\infty} \gamma_{n} \sum_{0 \leq 2 k \leq n} \sum_{j=0}^{M_{n-2 k}}\left\langle g, g_{k, j}^{n}\right\rangle_{L^{2}(Z)} f_{k, j}^{n} .
$$


Furthermore,

$$
f(x)=\sum_{n=0}^{\infty} \gamma_{n}^{-1} \sum_{0 \leq 2 k \leq n} \sum_{j=0}^{M_{n-2 k}}\left\langle g, g_{k, j}^{n}\right\rangle_{L^{2}(Z)} f_{k, j}^{n} .
$$

These equations are the realization of (5.1), (5.2) and (5.3) for the Radon transform. They are exactly the SVD derived in $[5,12]$, once the difference in notations is accounted for.

Below we derive the singular value decomposition using our notation here. We need a proposition that goes back to [17] when $d=2$.

Proposition 5.3. Let $P \in \mathcal{V}_{n}^{d}$. Then for each $t \in[-1,1]$ and $\xi \in S^{d-1}$,

$$
\mathcal{R} P(\xi, t)=b_{d-1}\left(1-t^{2}\right)^{\frac{d-1}{2}} \frac{C_{n}^{d / 2}(t)}{C_{n}^{d / 2}(1)} P(\xi) .
$$

In particular, the above formula applies to harmonic polynomials of degree $n$.

Proof. Let $Q_{\xi}$ be an orthogonal matrix whose first row is $\xi$. Then (3.1) shows that

$$
\mathcal{R} P(\xi, t)=\left(1-t^{2}\right)^{\frac{d-1}{2}} \int_{B^{d-1}} P\left(\left(t, \sqrt{1-t^{2}} y\right) Q_{\xi}\right) d y .
$$

The integral is a polynomial of $t$ since an odd power of $\sqrt{1-t^{2}}$ is always companioned by $y^{\alpha}$ with $|\alpha|$ being odd, which has integral zero. Therefore, $g(t)=$ $\left(1-t^{2}\right)^{-\frac{d-1}{2}} \mathcal{R} f(\xi, t)$ is of degree $k$ in $t$. Furthermore, the integral shows that

$$
g(1)=\operatorname{vol}\left(B^{d-1}\right) P(\xi)=b_{d-1} P(\xi) .
$$

If $g_{j} \in \Pi_{j}^{d}$ for $0 \leq j \leq n-1$, then the equation (3.2) and the fact that $P \in \mathcal{V}_{n}^{d}$ lead to

$$
\int_{-1}^{1} g(t) g_{j}(t)\left(1-t^{2}\right)^{\frac{d-1}{2}} d t=\int_{B^{d}} P(x) g_{j}(\langle x, \xi\rangle) d x=0,
$$

which shows immediately that the polynomial $g(t)$ is an orthogonal polynomial with respect to $\left(1-t^{2}\right)^{\frac{d-1}{2}}$ on $[-1,1]$, that is,

$$
g(t)=\left(1-t^{2}\right)^{-\frac{d-1}{2}} \mathcal{R} f(\xi, t)=a C_{n}^{d / 2}(t) .
$$

Setting $t=1$ determines the constant $a$ and completes the proof. Finally, (2.4) with $k=0$ show that harmonic polynomials of degree $n$ are in $\mathcal{V}_{n}^{d}$.

Corollary 5.4. Let $f_{k, j}^{n}$ be the orthonormal basis of $\mathcal{V}_{n}^{d}$ given in (2.4). Then

$$
\mathcal{R} f_{k, j}^{n}(\xi, t)=\gamma_{n} g_{k, j}^{n}(\xi, t)
$$

where the singular values $\gamma_{n}$ of $\mathcal{R} f$ are given by

$$
\gamma_{n}=b_{d-1} \sqrt{n ! /(d)_{n}} .
$$

Proof. Using (3.5) and (5.4), the equation (5.8) shows

$$
\mathcal{R} f_{k, j}^{n}(\xi, t)=b_{d-1}\left[h_{n}^{(d / 2)}\right]^{1 / 2}\left(1-t^{2}\right)^{\frac{d-1}{2}} \frac{C_{n}^{d / 2}(t)}{C_{n}^{d / 2}(1)}=\gamma_{n} g_{k, j}^{n}(\xi, t),
$$

where $\gamma_{n}=b_{d-1}\left[h_{n}^{(d / 2)}\right]^{-1 / 2} H_{n} / C_{n}^{(d / 2)}(1)$, which can be simplified by using (2.2), (3.6) and the fact that $C_{n}^{(d / 2)}(1)=(d)_{n} / n$ !. 
Theorem 5.5. The singular decomposition of $\mathcal{R} f$ satisfies

$$
\mathcal{R} f=c_{d / 2}\left(1-s^{2}\right)^{\frac{d-1}{2}} \sum_{n=0}^{\infty}\left[h_{n}^{(d / 2)}\right]^{-1} \int_{B^{d}} f(x) C_{n}^{d / 2}(\langle x, \xi\rangle) d x C_{n}^{d / 2}(t),
$$

where $M_{m}=\operatorname{dim} \mathcal{H}_{m}^{d}, c_{d / 2}$ is defined at (2.2); and

$$
\mathcal{R}^{*} g=c \sum_{n=0}^{\infty} \gamma_{n}\left[h_{n}^{(d / 2)}\right]^{-1} \int_{-1}^{1} \int_{S^{d-1}} \mathcal{R} f(\xi, t) C_{n}^{d / 2}(\langle x, \xi\rangle) C_{n}^{d / 2}(t) d \omega(\xi) d t
$$

where $c=c_{d / 2} b_{d-1} \sigma_{d}^{-1}$.

Proof. To prove (5.10), we note that by (3.5),

$$
f_{k, j}^{n}(x) g_{k, j}^{n}(\xi, t)=\left[h_{n}^{(d / 2)}\right]^{-1 / 2} H_{n}^{-1}\left(1-t^{2}\right)^{\frac{d-1}{2}} C_{n}^{d / 2}(t) f_{k, j}^{n}(x) f_{k, j}^{n}(\xi) .
$$

Since the constants are independent of $k$ and $j$, we can use (2.7) to write the summations in $k$ and $j$ of (5.10) in a compact form. Collecting constants and using (3.6), (5.9) and (2.2), we easily verify that

$$
\gamma_{n}\left[h_{n}^{(d / 2)}\right]^{-1 / 2} H_{n}^{-1} f_{k, j}^{n}(x) \frac{n+d / 2}{d / 2}=b_{d-1}\left[h_{n}^{(d / 2)}\right]^{-1} .
$$

Finally we note that $b_{d-1} b_{d}^{-1}=c_{d / 2}$. The proof of (5.11) is similar.

It is worth to comment that the two expressions (5.10) and (5.11) are independent of the choice of orthonormal bases, and the equation (2.7) implies that we can deduce the SVD from them using any orthonormal basis. In $[5,12]$, the SVD in terms of orthogonal basis with respect to $W_{\mu}$ is derived. In these more general cases, however, the simple analogue of the second equations of (5.5) and (5.6) do not hold. The reason again lies in the fact that the constant in (3.9) depends on $k$.

Finally, by (5.3), the truncation of the expansion of $f$ becomes

$$
S_{n}^{*} f(x)=\sum_{m=0}^{n} \gamma_{m}^{-1} \sum_{0 \leq 2 k \leq m} \sum_{j=0}^{M_{m-2 k}}\left\langle g, g_{k, j}^{m}\right\rangle_{L^{2}(Z)} f_{k, j}^{m} .
$$

Just as in the equations (5.5) and (5.6), we can use (5.12) and (2.7) to derive a compact formula. The formula, however, is exactly $S_{n} f$. As a consequence, we see the truncated SVD algorithm agrees with that formula (3.11). Hence, truncated SVD can be effectively implemented by using the OPED algorithm.

\section{REFERENCES}

[1] B. Bojanov and I. K. Georgieva, Interpolation by bivariate polynomials based on Radon projections, Studia Math, 162 (2004), 141 - 160.

[2] T. Bortfeld and U. Oelfke, Fast and exact 2D image reconstruction by means of Chebyshev decomposition and backprojection, Phys. Med. Biol.. 44 (1999), 1105-1120.

[3] A. Caponnetto and M. Bertero, Tomography with a finite set of projections: singular value decomposition and resolution, Inverse Problem, 13 (1997), 1191-1205.

[4] A. M. Cormack, Representation of a function by its line integrals with some radiological applications, J. Appl. Phys, 35 (1964), 2908-13.

[5] M. E. Davison, A singular value decomposition for the Radon transform in $n$-dimensional Euclidean space, Numer. Func. Anal. and Optimiz., 3 (1981), 321-340.

[6] M. E. Davison and F. A. Grünbaum, Tomographic reconstruction with arbitrary directions, Comm. Pure Appl. Math. 34 (1981), 77-119.

[7] C. F. Dunkl and Yuan Xu, Orthogonal polynomials of several variables, Cambridge Univ. Press, 2001. 
[8] S. Helgason, The Radon transform, Birkhäuser, Basel and Boston, 1980.

[9] G. T. Herman, Image reconstruction from projections, Academic Press, New York, 1980.

[10] A. C. Kak and M. Slaney, Principles of computerized tomographic imaging, IEEE Press, New York, 1988; Reprint as Classics in Applied Mathematics, 33. SIAM, Philadelphia, PA, 2001.

[11] B. Logan and L. Shepp, Optimal reconstruction of a function from its projections, Duke Math. J. 42 (1975), 645-659.

[12] A. Louis, Orthogonal function series expansions and the null space of the Radon transform, SIAM J. Math. Anal., 15 (1984), 621- 633.

[13] A. Louis, Incomplete data problems in x-ray computerized tomography I. Singular value decomposition of the limited angle transform Numer. Math. 48 (1986), 251-262.

[14] P. Maass, The x-ray transform: singular value decomposition and resolution, Inverse Problem, 3 (1987), 729-741.

[15] W. R. Madych, Summability and approximate reconstruction from Radon transform data, Contemporary Mathematics, Vol. 113 (1990), 189-219.

[16] W. R. Madych and S. A. Nelson, Polynomial based algorithms for computed tomography, SIAM J. Appl. Math. 43 (1983), 157-185.

[17] R. Marr, On the reconstruction of a function on a circular domain from a sampling of its line integrals, J. Math. Anal. Appl., 45 (1974), 357-374.

[18] I. P. Mysovskikh, Interpolatory cubature formulas, (in Russian), "Nauka", Moscow, 1981.

[19] F. Natterer, The mathematics of computerized tomography, Reprint of the 1986 original. Classics in Applied Mathematics, 32. SIAM, Philadelphia, PA, 2001.

[20] P. Petrushev, Approximation by ridge functions and neural networks, SIAM J. Math. Anal. 30 (1999), 155-189.

[21] A. Stroud, Approximate calculation of multiple integrals, Prentice Hall, Englewood Cliffs, NJ, 1971.

[22] B. Sündermann, On projection constants of polynomial space on the unit ball in several variables, Math. Z. 188 (1984), 111-117.

[23] G. Szegő, Orthogonal Polynomials, Amer. Math. Soc. Colloq. Publ. Vol.23, Providence, 4th edition, 1975.

[24] Yuan Xu, Summability of Fourier orthogonal series for Jacobi weight on a ball in $\mathbb{R}^{d}$, Trans. Amer. Math. Soc. 351 (1999), 2439-2458.

[25] Yuan Xu, Representation of reproducing kernels and the Lebesgue constants on the ball, $J$. Approx. Theory 112 (2001), 295-310.

[26] Yuan Xu, Weighted approximation of functions on the unit sphere, Constructive Approx. 21 (2005), 1-28.

[27] Yuan $\mathrm{Xu}, \mathrm{A}$ new approach for reconstruction of images from Radon projections, Adv. in Applied Math., 36 (2006), 388-420.

[28] Yuan Xu and O. Tischenko, Fast OPED algorithm for reconstruction of images from Radon data, submitted, 2006. arXiv:math/0703617.

[29] Yuan Xu, O. Tischenko and C. Hoeschen, A new reconstruction algorithm for Radon Data, Proc. SPIE, Medical Imaging 2006: Physics of Medical Imaging, vol. 6142 (2006), p. 791798.

Department of Mathematics University of Oregon Eugene, Oregon 97403-1222.

E-mail address: yuan@math.uoregon.edu 